\title{
Emerging role of rasburicase in the management of increased plasma uric acid levels in patients with hematologic malignancies
}

This article was published in the following Dove Press journal:

Journal of Blood Medicine

3 February 2011

Number of times this article has been viewed

\author{
LeAnne D Kennedy' \\ Susannah Koontz ${ }^{2}$ \\ Kamakshi Rao ${ }^{3}$ \\ 'Wake Forest University Baptist \\ Medical Center, Winston Salem, NC, \\ USA; ${ }^{2}$ Koontz Oncology Consulting \\ LLC, Houston, TX, USA; ${ }^{3}$ UNC \\ Hospitals, Chapel Hill, NC, USA
}

\begin{abstract}
Tumor lysis syndrome (TLS) is defined as a group of metabolic derangements that result from the massive and abrupt release of cellular components into the bloodstream after rapid lysis of tumor cells. Breakdown of released materials leads to a number of electrolyte abnormalities, including elevated uric acid concentrations in the blood (hyperuricemia), which carries potentially serious consequences. The diagnosis, prevention, and management of TLS is complicated by variability in definitions, differences in risk factors based on patient- and tumorspecific characteristics, and practitioner preferences in terms of pharmaceutical management strategies. The best prevention and management option for a particular patient depends on the patient's baseline risk for TLS development, the severity of symptoms in the event of TLS development, practical management considerations, and financial implications of treatment.

Keywords: tumor lysis syndrome, uric acid, rasburicase
\end{abstract}

\section{Epidemiology}

The incidence of tumor lysis syndrome (TLS) varies with the type of tumor, but TLS occurs most frequently ${ }^{1}$ in patients with hematologic malignancies such as acute lymphoblastic leukemia (5\%-26\%), acute myelogenous leukemia (3\%-17\%), Burkitt's lymphoma (27\%), and high-grade non-Hodgkin's lymphoma (other than Burkitt's type; up to $42 \%){ }^{1-5}$ TLS is observed most commonly after the initiation of cytotoxic treatments, cytolytic antibodies, and radiation therapies, but it can also occur spontaneously before antitumor therapy is administered. ${ }^{3}$ Malignancies with characteristics such as a rapid proliferative rate, a large tumor burden, and high sensitivity to chemotherapy increase the risk for TLS., ${ }^{1,2}$ Other patient-specific characteristics associated with increased risk for developing TLS include pre-existing hyperuricemia, renal dysfunction, and hypovolemia. ${ }^{1}$

\section{Etiology and pathophysiology}

The metabolic disturbances that occur with TLS are due primarily to the release of intracellular metabolites such as nucleic acids, proteins, peptides, phosphate, and potassium during cell lysis..$^{1,2}$ The release of these metabolites in large amounts overloads the renal system, leading to hyperkalemia, hyperphosphatemia, hypocalcemia (as a downstream effect of hyperphosphatemia), uremia, acidosis, and hyperuricemia. ${ }^{1,2}$

Hyperuricemia and its associated complications are the most frequently recognized manifestations of TLS and can lead to many other clinical consequences. ${ }^{2}$ Increases in serum uric acid concentrations result from the release and subsequent breakdown of
Wake Forest University Baptist Medical Center,Winston-Salem, NC, USA

Email lakenned@wfubmc.edu 
purine-containing nucleic acids from cells. Purine catabolism is a multistep process by which adenine and guanine are broken down and eventually excreted. The enzyme xanthine oxidase converts hypoxanthine to xanthine in the pathway for adenine catabolism; it also converts xanthine to uric acid in the adenine and guanine catabolism pathways. In most organisms, uric acid is degraded further to allantoin through the action of urate oxidase. ${ }^{6}$ However, in humans, apes, and some other primates, urate oxidase has been rendered nonfunctional through mutations during evolution. ${ }^{6}$ This lack of functional urate oxidase leads to baseline concentrations of uric acid that are high, relative to those in other mammals. ${ }^{6}$ Under normal circumstances, uric acid concentrations can be maintained at acceptable levels by renal excretion. However, when excretion cannot keep up with production and the concentration of uric acid rises, it may become insoluble and form crystals. Crystallization of uric acid in the renal tubules can cause renal insufficiency that may progress to acute renal failure. ${ }^{1}$

\section{Patient presentation}

Clinical signs of TLS may include a wide range of symptoms, from mild (nausea, vomiting, diarrhea, lethargy, edema) to very serious and life threatening (cardiac dysrhythmias, congestive heart failure, tetany, syncope, seizure, and sudden death). ${ }^{1,3}$ Although the set of metabolic abnormalities that constitute TLS generally has been long established, systematic classification has been attempted only recently. In 2004, Cairo and Bishop ${ }^{3}$ proposed dividing the diagnosis of TLS into the subcategories of laboratory TLS and clinical TLS, based in part on earlier work by Hande and Garrow. ${ }^{4}$ The diagnosis of laboratory TLS is based solely on the occurrence of electrolyte and metabolic abnormalities, whereas clinical TLS includes the same abnormalities, but also takes into account the physical manifestations and consequences of active TLS. These Cairo-Bishop definitions, which have application to both pediatric and adult patients, were integrated into the latest guidelines for the prevention and treatment of TLS, which were published in June 2008. ${ }^{1}$

\section{Common staging systems}

Most of the complications of TLS can be avoided through appropriate preventive measures or readily managed if recognized early; otherwise, serious and fatal consequences may occur. Therefore, it is important for clinicians to be cognizant of strategies to identify patients who are at risk before initiating chemotherapy. ${ }^{1,2}$ A grading system ${ }^{3}$ based on the presence or absence of clinical and laboratory criteria has been developed for TLS to aid in the stratification of patients according to risk and to help identify appropriate prophylaxis and treatment for patients at risk for or with established TLS. ${ }^{1,2}$ Currently, this grading system is being prospectively evaluated in pediatric patients being treated with chemoimmunotherapy and rasburicase for newly diagnosed advanced B-cell non-Hodgkin's lymphoma (as per the Children's Oncology Group Study ANHL01P1). ${ }^{3,7}$ More recently, a risk evaluation model has been proposed by Cairo et al that incorporates risk factors from prior models (eg, tumor type and extent and bulk of disease) as well as risk factors not previously addressed in earlier stratification schemes (eg, age, pre-existing renal dysfunction, and renal involvement). ${ }^{8}$ Fundamental approaches to the successful prevention and management of TLS include awareness of potential causes, identification and close monitoring of patients at high risk, implementation and optimal use of appropriate prophylactic measures, vigilant monitoring of laboratory and electrolyte levels, watching for signs and symptoms of TLS in patients at risk, and initiation of more active treatments when necessary. Early recognition and treatment for metabolic abnormalities, with the help of the grading system, can usually prevent the severe and life-threatening consequences that may occur with TLS (see Table 1). ${ }^{1,2}$

\section{Treatment}

Management strategies for TLS include continuation of preventive measures (ie, hydration, allopurinol use), correction of fluid overload or dehydration, and treatment for electrolyte, laboratory, and acid-based abnormalities. Pharmacologic agents used to treat patients with TLS-related hyperuricemia include allopurinol, uricozyme, and rasburicase.

\section{Hydration and fluid management}

Vigorous hydration and diuresis are essential for the prevention and management of TLS. Generally, patients are hydrated with approximately $3 \mathrm{~L} / \mathrm{m}^{2} / \mathrm{d}$ of fluid (or $0.2 \mathrm{~L} / \mathrm{kg} / \mathrm{d}$ if the patient weighs $\leq 10 \mathrm{~kg}$ ); urine output should be maintained at $\geq 100 \mathrm{~mL} / \mathrm{m}^{2} / \mathrm{h}$ (or $\geq 3 \mathrm{~mL} / \mathrm{kg} / \mathrm{h}$ if the patient weighs $\leq 10 \mathrm{~kg}) .{ }^{3}$ Hydration and enhanced urine flow aid in the excretion of uric acid and phosphate by increasing intravascular volume, renal blood flow, and glomerular filtration rate. Diuretics may be used to maintain adequate urine output if sufficient diuresis is not achieved with intravenous hydration alone. However, the use of diuretics is contraindicated in the setting of hypovolemia or obstructive uropathy. In the past, sodium bicarbonate was used commonly to alkalinize the urine to improve the solubility of uric acid and 
Table I TLS Risk assessment and recommended therapy

\begin{tabular}{lll}
\hline Degree of risk & Tumor type & Therapy recommended \\
\hline High & $*$ Burkitt's lymphoma & Monitoring \\
& $*$ High-grade non-Hodgkin's lymphoma & Hydration \\
& $*$ Lymphoblastic leukemia & Rasburicase \\
& $*$ T-cell acute leukemia & Monitoring \\
& $*$ Acute myeloid leukemia with high white blood cell count & Hydration \\
& $*$ Low-grade lymphoma treated with definitive therapy & Allopurinol \\
& $*$ Multiple myeloma & \\
& $*$ Breast carcinoma treated with chemotherapy/hormonal therapy & \\
& $*$ Small-cell lung carcinoma & Monitoring \\
& $*$ Germ-cell tumors (seminoma, ovarian) & Hydration $+/-$ allopurinol \\
& $*$ Neuroblastoma & \\
& $*$ Hodgkin's lymphoma & \\
& $*$ Low-grade lymphoma treated with interferon & \\
& $*$ Medulloblastoma &
\end{tabular}

promote its excretion. On the other hand, alkalinization does little to increase the solubility of xanthine and hypoxanthine. Alkalinization is no longer recommended, because of equivocal evidence of efficacy and potential complications, including induction of inadvertent metabolic alkalosis and obstructive uropathies caused by precipitation of calcium phosphate and xanthine crystals in the renal tubules. ${ }^{1,2,9-11}$

\section{Inhibition of uric acid production}

Allopurinol, a potent xanthine oxidase inhibitor, blocks conversion of the purine metabolites hypoxanthine and xanthine to uric acid. Allopurinol has been shown to be effective in reducing serum uric acid concentrations ${ }^{12-14}$ and the incidence of uric acid-related obstructive uropathy ${ }^{14}$ in patients who are at risk for developing TLS. By reducing formation of uric acid, allopurinol decreases the incidence of obstructive uropathy caused by crystallization of uric acid. Although allopurinol is effective in preventing de novo formation of uric acid, it does not reduce the amount of uric acid already present. Therefore, it can be used concurrently with rasburicase and should be initiated prophylactically $0.5-3$ days before administration of cytotoxic therapy. Allopurinol is available in both oral and intravenous dosage forms. The recommended daily dose is $300 \mathrm{mg}$ (pediatric oral dose: $50-100 \mathrm{mg} / \mathrm{m}^{2}$ every 8 hours [maximum dose $300 \mathrm{mg} / \mathrm{m}^{2} / \mathrm{d}$ ] or $10 \mathrm{mg} / \mathrm{kg} / \mathrm{d}$ divided every 8 hours [maximum dose $800 \mathrm{mg} / \mathrm{d}$ ]; pediatric intravenous dose: $200-400 \mathrm{mg} / \mathrm{m}^{2} / \mathrm{d}$ in 1-3 divided doses [maximum dose $600 \mathrm{mg} / \mathrm{d}]$ ), but dosage adjustments must be made in patients with glomerular filtration rate $<50 \mathrm{~mL} / \mathrm{min}^{1,3}$ Allopurinol is well tolerated but may be associated with nausea, diarrhea, and rash. ${ }^{9}$ Allopurinol also may interfere with the metabolism of 6-mercaptopurine and azathioprine, requiring that these medications be administered at reduced doses. Until recently, allopurinol had been the only xanthine oxidase inhibitor available. However in February 2009, the US Food and Drug Administration (FDA) approved febuxostat for the chronic management of hyperuricemia in patients with gout. However, no data currently exist on its efficacy in the management of hyperuricemia-related TLS.

\section{Enzymatic removal of uric acid}

Because allopurinol is effective only in preventing further formation of uric acid, an alternative option for the treatment of hyperuricemia is to promote the breakdown of uric acid to a more soluble form. ${ }^{2}$ Uricozyme, a nonrecombinant preparation of urate oxidase enzyme, facilitates the conversion of uric acid to allantoin, which is 5-10 times more soluble in urine than uric acid is. ${ }^{1}$ Urate oxidase enzyme is not present in humans ${ }^{6}$ and is therefore isolated from the fungus Aspergillus flavus. ${ }^{1}$ Rasburicase is a recombinant, highly purified urate oxidase enzyme approved in 2002 by the FDA for the initial management of hyperuricemia in pediatric patients with leukemia, lymphoma, and solid tumor malignancies who are receiving chemotherapy that is expected to result in TLS and hyperuricemia. In 2009, the indications for rasburicase were expanded to include adult patients with the same clinical scenarios as those described in children. In Europe, rasburicase is indicated for use in children and adults with hematologic malignancy.

Rasburicase has been shown to be effective and safe in reducing hyperuricemia in patients who are at risk for development of TLS. ${ }^{15-17}$ Rasburicase is generally well tolerated; in a study of 1069 patients, only 44 (4\%) reported an adverse reaction that was judged to be related 
to rasburicase. ${ }^{23}$ Mild adverse events include, fever, nausea, vomiting, constipation, and abdominal pain. In rare instances, rasburicase treatment can cause serious adverse reactions, including anaphylaxis, hemolysis, methemoglobinemia, sepsis, and rash. It is contraindicated in patients with glucose-6-phosphate dehydrogenase (G6PD) deficiency. In contrast to allopurinol, rasburicase doses do not need to be adjusted in patients with renal impairment; therefore, it may be given without dose adjustment to patients with renal insufficiency. Per drug interactions, rasburicase does not interfere with activity of the cytochrome P450 system and should not alter drug metabolism by those enzymes.

Rasburicase may interfere with the accurate measurement of plasma uric acid concentrations because it continues to degrade uric acid in blood samples after they have been drawn from patients. Therefore, blood samples should be drawn in chilled heparin tubes and kept on ice, starting immediately after collection. All uric acid assays should be completed within 4 hours. The half-life of rasburicase is approximately 16-21 hours, depending on the dose; ${ }^{1}$ after appropriate sample collection, uric acid concentrations should be monitored closely for 3-4 days after the final dose of rasburicase is administered during a given course of treatment. If these assay procedures are not followed, uric acid assay concentrations may appear erroneously low, which could prompt premature discontinuation of rasburicase therapy with a consequent rebound in uric acid levels and return of the risk potential for TLS for the patient.

The dosage of rasburicase recommended by the FDA is $0.2 \mathrm{mg} / \mathrm{kg}$ once daily in $50 \mathrm{~mL}$ of normal saline solution, given as an intravenous infusion over 30 minutes for up to 5 days. ${ }^{1}$ This recommended dosage, recently modified from the original suggested dosage range of $0.15-0.2 \mathrm{mg} / \mathrm{kg}$ daily for 5 days, was based on clinical studies that led to approval of rasburicase for its current indication. Subsequently, data from several other studies have shown that rasburicase can be clinically effective when administered at lower doses and for shorter durations. ${ }^{1,2}$

Various studies have used weight-based rasburicase dosing with a duration that could be varied according to the judgment of the investigator. In a compassionate-use study in 1069 patients (996 patients were evaluable for efficacy, of which 658 were children and 338 were adults), a median of three doses (range 1-14 doses) of rasburicase were given per patient with a median treatment duration of 3 days (range 1-12 days); $99 \%$ of patients showed a positive response to treatment. ${ }^{18}$ In another study in 100 adult patients with diffuse large B-cell lymphoma who were at risk for developing TLS, including 11 patients who presented with elevated uric acid concentration before chemotherapy, those who received rasburicase $0.2 \mathrm{mg} / \mathrm{kg} / \mathrm{d}$ for $\geq 3$ days experienced rapid and dramatic decreases in uric acid levels. ${ }^{18}$ In a retrospective, single-center study of six adults and two children with hematologic malignancies and uric acid concentrations $\geq 8 \mathrm{mg} / \mathrm{dL}$ ( $\geq 473 \mu \mathrm{mol} / \mathrm{L}$ ), patients received only a single dose of rasburicase of about $0.15 \mathrm{mg} / \mathrm{kg}$ based on actual weight if they were not obese, and based on an adjusted weight if they were obese. ${ }^{20}$ This single dose of rasburicase was effective in lowering uric acid concentrations and maintaining those concentrations within normal limits for the next 96 hours in this small study. ${ }^{20}$ Although four patients had impaired renal function at baseline, two of whom met criteria for TLS, no patient required hemodialysis. ${ }^{20}$ However, in two other studies, a substantial percentage of patients (5 of 13 [38\%] and 27 of 40 [68\%]) required multiple doses of rasburicase to successfully treat hyperuricemia..$^{21,22}$ Moreover, Wang et al $(n=45)$ found that the median number of required doses of rasburicase (given at a dose of $0.2 \mathrm{mg} / \mathrm{kg}$ ) was three in children and four in adults. ${ }^{23}$ Finally, a case study on one obese patient suggested that a single-dose strategy based on ideal body weight may be as effective as one based on actual body weight. ${ }^{24}$

Several small studies have explored the use of fixed instead of weight-adjusted doses in adult and pediatric patients, including the use of lower than recommended doses and of fewer than recommended administrations. In a study of 11 adult patients with hematologic malignancies, McDonnell et al reported that $6 \mathrm{mg}$ of rasburicase was effective in lowering uric acid concentrations in 10 of 11 patients. ${ }^{25}$ However, in one morbidly obese patient who weighed $259 \mathrm{~kg}$, the uric acid concentration decreased only from $17.4 \mathrm{mg} / \mathrm{dL}(1030 \mu \mathrm{mol} / \mathrm{L})$ to $15.4 \mathrm{mg} / \mathrm{dL}(911 \mu \mathrm{mol} / \mathrm{L})$ after the first dose, and a second dose of $12 \mathrm{mg}(0.05 \mathrm{mg} / \mathrm{kg})$ was necessary to reduce the uric acid concentration to $1.4 \mathrm{mg} / \mathrm{dL}(83 \mu \mathrm{mol} / \mathrm{L}) .{ }^{25}$ Similarly, Hutcherson et al reported that 1 of 11 patients who received a single dose of rasburicase $6 \mathrm{mg}$ experienced inadequate uric acid reduction and required a second $6 \mathrm{mg}$ dose.${ }^{26}$ Reeves et al prospectively compared the efficacy of a fixed $7.5 \mathrm{mg}$ dose of rasburicase and weight-based dosing at $0.15 \mathrm{mg} / \mathrm{kg}$ in 40 adult patients. Normalization of uric acid concentrations was achieved with both treatments and followed a similar time course. ${ }^{27}$ An even lower dose ( $3 \mathrm{mg}$ ) was studied by Trifilio et al in 43 adult patients; uric acid normalization was achieved after 48 hours, but six patients (14\%) required a second dose (rasburicase $3 \mathrm{mg}$ or $1.5 \mathrm{mg}$ ) 24 hours after administration of the first 
$3 \mathrm{mg}$ dose to resolve hyperuricemia completely. ${ }^{28}$ Lee et al reported rapid normalization of serum uric acid concentrations as a result of single administration of a fixed dose of rasburicase $4.5 \mathrm{mg}$ in three children (aged 4-13 years) with acute lymphoblastic leukemia. Weight-based doses of rasburicase ranged from 0.08 to $0.26 \mathrm{mg} / \mathrm{kg} .{ }^{29}$

At least some of the off-label treatment approaches using reduced doses or less frequent than recommended administration of rasburicase appeared to be motivated in part by the desire to minimize the substantial costs associated with rasburicase therapy. ${ }^{25-29}$ Based on its current average sales price, a single $6 \mathrm{mg}$ dose of rasburicase would cost about US\$1990, ${ }^{29}$ which is much less than the approximately US\$21,000 cost of the FDA-approved regimen ( 5 days at $0.15 \mathrm{mg} / \mathrm{kg}$, based on an average total body weight of $85 \mathrm{~kg}$ ). Further investigation is warranted to determine the efficacy of single-dose rasburicase for the prevention or treatment of hyperuricemia associated with TLS. However, because the cost of extended hospitalization and of hemodialysis in patients with hematologic malignancies who develop renal impairment have been shown to total up to US\$52,000 (2002), ${ }^{30}$ even administration of the full course of rasburicase in appropriately selected patients may result in substantial cost savings. In the interim, expert practice requires administration of the number of doses necessary to maintain plasma uric acid concentration within a clinically acceptable range. Actual values are not defined in the guidelines. ${ }^{1}$

The development of rasburicase may have reduced the incidence and severity of TLS and its associated complications in patients receiving cytolytic therapy for hematologic malignancies. However, no data on outcomes (eg, acute kidney injury, renal failure, hemodialysis, death) have been published since this agent was developed. Future studies are needed to examine clinical outcomes with the use of rasburicase in the prevention and treatment of hyperuricemia associated with TLS in adult and pediatric hematology and oncology patients. Pharmacoeconomic studies are also warranted to determine the optimal and most cost-effective dosing strategy for rasburicase use.

\section{Summary}

TLS is a dangerous complication of chemotherapeutic intervention that is most common in patients with hematologic malignancies. Preventive measures should be used to reduce the incidence of TLS in patients who are at risk. If TLS-associated hyperuricemia develops despite preventive efforts, rasburicase treatment is an effective, well-tolerated method for returning uric acid concentrations to acceptable levels. Rasburicase has a special role in therapy for patients at high risk for TLS, such as those with acute lymphocytic leukemia and highly aggressive lymphomas, especially if the tumor burden is high. Administration of a reduced number or quantity of rasburicase, whether given as weight-based or flat doses, may help to prevent TLS complications without incurring the cost of the recommended 5-day treatment course.

\section{Disclosure}

The authors report no conflicts of interest in this work.

\section{References}

1. Coiffier B, Altman A, Pui CH, Younes A, Cairo MS. Guidelines for the management of pediatric and adult tumor lysis syndrome: an evidencebased review. J Clin Oncol. 2008;26:2767-2778.

2. Hochberg J, Cairo MS. Tumor lysis syndrome: current perspective. Haematologica. 2008;93:9-13.

3. Cairo MS, Bishop M. Tumour lysis syndrome: new therapeutic strategies and classification. Br J Haematol. 2004;127:3-11.

4. Hande KR, Garrow GC. Acute tumor lysis syndrome in patients with high-grade non-Hodgkin's lymphoma. Am J Med. 1993;94:133-139.

5. Wossmann W, Schrappe M, Meyer U, Zimmermann M, Reiter A. Incidence of tumor lysis syndrome in children with advanced stage Brukitt's lymphoma/leukemia before and after introduction of prophylactic use of urate oxidase. Ann Hematol. 2003;82:160-165.

6. Oda M, Satta Y, Takenaka O, Takahata N. Loss of urate oxidase activity in hominoids and its evolutionary implications. Mol Biol Evol. 2002; 19:640-653.

7. Goldman S, Lynch J, Harrison L, et al. Preliminary results of the addition of rasburicase to the reduction cycle and rituximab to the induction and consolidation cycles of FAB group C chemotherapy in children and adolescents with advanced stage (bone marrow \pm CNS) mature B-cell non-Hodgkin lymphoma (B-NHL): a Children's Oncology Group report. Blood. 2009;114:104.

8. Cairo MS, Coiffier B, Reiter A, Younes A. Recommendations for the evaluation of risk and prophylaxis of tumour lysis syndrome (TLS) in adults and children with malignant diseases: an expert TLS panel consensus. Br J Haematol. 2010;149:578-586.

9. Ten Harkel ADJ, Kist-van Holthe JE, van Weel M, van der Vorst MMJ. Alkalinization and the tumor lysis syndrome. Med Pediatr Oncol. 1998; 31:27-28.

10. Chantada GL, Sackmann-Muriel F. Alkalinization and tumor lysis syndrome. Med Pediatr Oncol. 1999;32:156.

11. Van den Berg H, Reintsema AM. Renal tubular damage in rasburicase: risks of alkalinisation. Ann Oncol. 2004;15:175-176.

12. DeConti RC, Calabresi P. Use of allopurinol for prevention and control of hyperuricemia in patients with neoplastic disease. $N$ Engl J Med 1966;274:481-486.

13. Krakoff IH, Meyer RL. Prevention of hyperuricemia in leukemia and lymphoma: use of allopurinol, a xanthine oxidase inhibitor. JAMA. 1965;193:1-6

14. Smalley RV, Guaspari A, Haase-Statz S, Anderson SA, Cederberg D, Hohneker JA. Allopurinol: intravenous use for prevention and treatment of hyperuricemia. J Clin Oncol. 2000;18:1758-1763.

15. Allopurinol [prescribing information]. Corona, CA: Watson Laboratories, Inc.; 2006.

16. Uloric [prescribing information]. Deerfield, IL: Takeda Pharmaceuticals US; 2009.

17. Elitek [prescribing information]. Bridgewater, NJ: sanofi-aventis US; 2010.

18. Data on file. Bridgewater, NJ: sanofi-aventis US. 
19. Fasturtec [summary of product characteristics]. London, UK: European Medicines Agency; 2008.

20. Goldman SC, Holcenberg JS, Finklestein JZ, et al. A randomized comparison between rasburicase and allopurinol in children with lymphoma or leukemia at high risk for tumor lysis. Blood. 2001;97:2998-3003.

21. Pui CH, Mahmoud HH, Wiley JM, et al. Recombinant urate oxidase for the prophylaxis or treatment of hyperuricemia in patients with leukemia or lymphoma. J Clin Oncol. 2001;19:697-704.

22. Coiffier B, Mounier N, Bologna S, et al. Efficacy and safety of rasburicase (recombinant urate oxidase) for the prevention and treatment of hyperuricemia during induction chemotherapy of aggressive nonHodgkin's lymphoma: results of the GRAAL1 (Groupe d'Etude des Lymphomes de l'Adulte Trial on Rasburicase Activity in Adult Lymphoma) study. J Clin Oncol. 2003;21:4402-4406.

23. Jeha S, Kantarjian H, Irwin D, et al. Efficacy and safety of rasburicase, a recombinant urate oxidase (Elitek), in the management of malignancyassociated hyperuricemia in pediatric and adult patients: final results of a multicenter compassionate use trial. Leukemia. 2005;19:34-38.

24. Liu CY, Sims-McCallum RP, Schiffer CA. A single dose of rasburicase is sufficient for the treatment of hyperuricemia in patients receiving chemotherapy. Leuk Res. 2005;29:463-465.

25. Ho VQ, Wetzstein GA, Patterson SG, Bradbury R. Abbreviated rasburicase dosing for the prevention and treatment of hyperuricemia in adults at risk for tumor lysis syndrome. Support Cancer Ther. 2006;3: $178-182$.

26. Hunter HM, Rule SAJ. Rasburicase is effective in the prophylaxis of tumour lysis syndrome when used over a short duration in patients with haematological malignancy. Br J Haematol. 2005;129(Suppl 1):42.

27. Wang LY, Shih LY, Chang H, et al. Recombinant urate oxidase (rasburicase) for the prevention and treatment of tumor lysis syndrome in patients with hematologic malignancies. Acta Haematol. 2006;115: $35-38$.
28. Arnold TM, Reuter JP, Delman BS, Shanholtz CB. Use of single-dose rasburicase in an obese female. Ann Pharmacother. 2004;38: 1428-1431.

29. McDonnell AM, Lenz KL, Frei-Lahr DA, Hayslip J, Hall PD. Single-dose rasburicase $6 \mathrm{mg}$ in the management of tumor lysis syndrome in adults. Pharmacotherapy. 2006;26:806-812.

30. Hutcherson DA, Gammon DC, Bhatt MS, Faneuf M. Reduced-dose rasburicase in the treatment of adults with hyperuricemia associated with malignancy. Pharmacotherapy. 2006;26:242-247.

31. Reeves DJ, Bestul DJ. Evaluation of a single fixed dose of rasburicase $7.5 \mathrm{mg}$ for the treatment of hyperuricemia in adults with cancer. Pharmacotherapy. 2008;28:685-690.

32. Trifilio S, Gordon L, Singhal S, et al. Reduced-dose rasburicase (recombinant xanthine oxidase) in adult cancer patients with hyperuricemia. Bone Marrow Transplant. 2006;37:997-1001.

33. Lee AC, Li CH, So KT, Chan R. Treatment of impending tumor lysis with single-dose rasburicase. Ann Pharmacother. 2003;37:1614-1617.

34. Centers for Medicare and Medicaid Services. 2009 Average Sales Price Drug Pricing Files. Available from: http://www.cms.hhs. gov/apps/ama/license.asp?file=/McrPartBDrugAvgSalesPrice/ downloads/April_2009_ASP_Pricing_File_by_HCPCS.zip. Accessed Mar 232009

35. Bell T, Candrilli S, Irish W, Morris E, Goldman SC, Cairo MS. Acute renal failure (ARF) with or without dialysis (D) is associated with a significant increase in mean length of stay (LOS) and total costs (TC) in patients hospitalized with acute leukemia (AL) and/or lymphoma (L). Proc Am Soc Clin Oncol. 2003;22(Abstr 2195).
Journal of Blood Medicine

\section{Publish your work in this journal}

The Journal of Blood Medicine is an international, peer-reviewed, open access, online journal publishing laboratory, experimental and clinical aspects of all topics pertaining to blood based medicine including but not limited to: Transfusion Medicine; Blood collection, Donor issues, Transmittable diseases, and Blood banking logistics; Immunohematology; Artificial and

\section{Dovepress}

alternative blood based therapeutics; Hematology; Biotechnology/nanotechnology of blood related medicine; Legal aspects of blood medicine; Historical perspectives. The manuscript management system is completely online and includes a very quick and fair peer-review system. Visit http://www.dovepress. com/testimonials.php to read real quotes from published authors. 